\title{
江苏省稻瘟病菌的毒性多样性及水稻品种的抗病性
}

\author{
陆 凡 凡, $^{1,}$ 郑小波 ${ }^{1}$ 陈志谊 ${ }^{2}$ 刘永锋 $^{2}$ 王法明 $^{2} \quad$ 范永坚 $^{2}$ \\ 1 (南京农业大学农业部病虫监测与治理重点开放实验室, 南京 210095) \\ 2 (江苏省农业科学院植保所, 南京 210014)
}

\begin{abstract}
摘要: 在 13 个已知日本抗病基因品种上检测 1997 1999 年采集自江苏省吴江、赣榆、通州、高邮和宜兴等 5 个代 表地区的 324 个稻瘟病菌株的毒性, 结果可将上述菌株划分为 90 种毒性类型, 表明江苏省稻瘟病菌存在着丰富的 毒性多样性。毒性类型组成在地区间存在较大的差异, 并且随着时间的推移, 稻瘟病菌毒性类型组成有差异加大 的趋势。在已知抗病基因品种上测定江苏省稻瘟病菌的毒力, 结果显示, $P i^{-} k^{5} 、 P i^{-} t a 、 P i^{-} t a^{2}$ 和 $P i^{-}-s h$ 等抗病基因对 江苏省的稻瘟病菌的抗谱很窄, 而 $P i^{-} i 、 P i^{-} z 、 P i^{-} z^{t}$ 和 $P i^{-} b$ 等抗病基因的抗谱比较宽, 可作为抗源加以利用。用 6 个代表性毒性类型菌株接种江苏省 80 个水稻主栽品种和新育成品种, 品种抗性分析表明, 上述水稻品种中的籼稻 和杂交稻对江苏省稻瘟病菌具有较高的抗性, 而粳稻品种的抗性较差。上述研究结果为利用水稻品种抗性多样性 控制稻瘟病提供了依据。
\end{abstract}

关键词: 稻瘟病菌, 毒性,多样性, 水稻品种, 抗病性

中图分类号 : S435.111.2,S435.111. $4^{+}$文献标识码: A 文章编号 : 1005-0094(2001) 03-0201-06

\section{Pathotype diversity of Magnaporthe grisea and resistance of rice cultivars to the pathogen in Jiangsu Province}

\author{
LU Fan ${ }^{1,2}$, ZHEN G Xiao-Bo ${ }^{1}$, CHEN Zhi- $\mathrm{Yi}^{2}$, L IU Yong-Feng ${ }^{2}$, WANG Fa-Ming ${ }^{2}$, FAN Yong-Jian ${ }^{2}$ \\ 1 Key Lab of Monitoring and management of Plant Disease and Insects, Ministry of A griculture, \\ Nanjing A gricultral University, Nanjing 210095 \\ 2 Institute of Plant Protection, Jiangsu Academy of A gricultural Sciences, Nanjing 210014
}

\begin{abstract}
Three hundred and twenty-four isolates were obtained from rice blast specimens collected from five typical areas - Wujiang, Ganyu, Tongzhou, Gaoyou and Yixing in Jiangsu Province during $1997 \sim 1999$. The isolates were assessed against $13 \mathrm{~J}$ apanese cultivars for which resistance genes have been determined and were divided into 90 pathotypes. This indicated that complex virulence diversity exists in Magnaporthe grisea in Jiangsu Province. The pathotype composition were dissimilar in the five typical areas, and from $1997 \sim 1999$, the dissimilarity tended to increase. The analysis of virulence frequency indicated that the resistance genes $P i^{-} k^{s}, P i^{-} t a, P i^{-} t a^{2}$ and $P i^{-} s h$ had narrow resistance spectra to Magnaporthe grisea isolates tested in Jiangsu Province, while the genes $\mathrm{Pi}^{-} i, \mathrm{Pi}^{-} \mathrm{z}, \mathrm{Pi}^{-} \mathrm{z}^{t}$ and $P i^{-} b$ had wide resistance spectra to the pathogen and had value in rice breeding as resources of resistance. Inoculation with six strains that represent six typical pathotypes of Magnaporthe grisea on 80 major cultivars and newly-bred cultivars of Jiangsu Province showed that most of the Indica cultivars and hybrid rice cultivars tested were resistant and the Japonica rice cultivars were susceptible to the pathogen. The results provide a basis for use of resistance diversity of rice cultivars to control rice blast.
\end{abstract}

Key words: Magnaporthe grisea, virulence, diversity, rice cultivar, resistance

稻瘟病菌 (有性态: Magnaporthe grisea, 无性 态: Pyricularia grisea) 引起的稻瘟病是世界水稻生
产最具毁灭性的病害之一, 该病常年流行,造成极大 经济损失, 是水稻生产的重要限制因素 $(\mathrm{Ou}, 1985)$ 。

* 基金项目 :江苏省自然科学基金(B K99184)、教育部骨干教师基金和教育部跨世纪优秀人才培养计划基金资助。 
利用抗病品种是防治该病的主要措施之一。然而, 一个抗病品种往往大面积种植 $3 \sim 5$ 年后即丧失抗 性, 导致稻瘟病再次流行 (Ou, 1980;杜正文, 1991)。 关于水稻抗瘟性丧失的原因, 通常认为主要有两个: 一是由于所推广的抗病品种在鉴定篮选时所选用的 菌株不能代表其群体毒性组成特征, 以致大面积推 广后, 潜在的毒性小种便发展成为优势种群, 使品种 丧失抗性; 二是由于稻瘟病菌易发生变异, 当抗病品 种大面积种植后所产生的寄主选择压力可能导致病 原菌发生变异, 产生新的毒性类型 (Zeigler et al., 1994）。因此, 要提高水稻抗病品种选育的有效性并 延长其使用寿命, 加强对稻瘟病菌群体毒性类型组 成、毒性的遗传结构特征及其变化趋势进行分析十 分必要 (Mekwatanakarn et al. 2000)。

江苏省位于暖温带向亚热带的过渡区域,光热、 水源、土壤等资源为稻作发展提供了极为有利的条 件。江苏的多种生态类型决定了稻作类型的多样 性。早、中、晚熟的籼、粳、糯稻齐全, 早稻、中稻、晚 稻均有, 常规稻和杂交稻并存(杨立畑等, 1990)。因 此, 江苏省水稻品种中存在着丰富的抗性资源。对 江苏省水稻品种进行抗性分析, 可以为合理利用品 种资源控制稻瘟病危害提供科学依据。

作者用 13 个日本的已知抗病基因品种对 1997 ～1999 年江苏省稻瘟病菌毒性类型进行了分析, 并 对江苏省 80 个主栽和新育成品种的抗瘟性进行了 分析, 以期明确江苏省稻瘟病菌群体的毒性结构特 征及江苏省水稻品种的抗性状况, 为制定稻瘟病的 持久控制策略提供依据。

\section{1 材料和方法}

\section{1 稻瘟病标样采集及菌株分离}

从 $1997 \sim 1999$ 年, 每年 9 10 月份在江苏省五 大稻区的 5 个代表县市吴江市、宜兴市、通州市、高 邮市和赣榆市的不同水稻品种上采集稻瘟病标样 100 份以上, 分别记载品种名称、采集地点。将标样 上的稻瘟病斑部位剪下, 用无菌水浸泡 $6 \mathrm{~h}$ 左右, 再 用无菌水荡洗 3 次, 放入垫有薄层湿脱脂棉的培养 皿中, 置 $25^{\circ} \mathrm{C}$ 恒温箱内保湿培养 $24 \mathrm{~h}$ 。长出孢子 后, 选取无杂菌样本, 在显微镜下 (无菌条件下) 用挑 针挑取单孢置于 $2 \%$ 琼脂培养基上于 $25^{\circ} \mathrm{C}$ 下培养。 待长成菌落后, 移到 PDA 斜面培养基上于 $25^{\circ} \mathrm{C}$ 恒 温下培养。所获得的单孢株作为供本研究使用的菌 株。

\section{2 诱导供试菌株产狍与狍子县浮液配制}

挑方法 1.1 获得的单孢株新鲜培养的菌块于玉 米粉稻汁培养基平板 (直径 $9 \mathrm{~cm}$ ) 上,每皿挑 3 块, 每块大小约 $5 \mathrm{~mm} \times 5 \mathrm{~mm}$, 在 $25^{\circ} \mathrm{C}$ 恒温下培养 7 10 天, 再用黑光灯连续照光 3 天促其产生狍子。待 大量产孢后, 用少量无菌水洗下孢子, 通过灭菌细纱 布过滤掉菌丝后, 配成狍子量约为 $5 \times 10^{4}$ 个 $/ \mathrm{mL}$ (100 倍显微镜, 每视野约 50 个孢子) 的孢子悬浮 液。玉米粉稻汁培养基配方为:玉米粉 $40 \mathrm{~g}$, 稻秆 $50 \mathrm{~g}$, 琼脂 $20 \mathrm{~g}$, 水 $1000 \mathrm{~mL}$ 。制作方法: 将 $50 \mathrm{~g}$ 稻 秆剪碎,加水 $1000 \mathrm{~mL}$ 煮沸 $30 \mathrm{~min}$, 用纱布滤去稻 秆, 冷却后加入 $40 \mathrm{~g}$ 玉米粉和 $20 \mathrm{~g}$ 琼脂混匀并加水 补足 $1000 \mathrm{~mL}$, 装瓶后高压灭菌 $\left(121^{\circ} \mathrm{C}, 30 \mathrm{~min}\right)$ 即 成。

\section{3 供试水稻品种及育苗}

13 个已知抗病基因的日本粳稻品种: 新 2 号 $\left[P i^{-} k^{s}, P i^{-} s h\right]$ 、爱知旭 $\left[P i^{-} a, P i^{-} 19(t)\right]$ 、藤坂 5 号 $\left[P i^{-} i, P i^{-} s h\right]$ 、草笛 $\left[\mathrm{Pi}^{-} k, P i^{-} s h\right]$ 、露明 $\left[\mathrm{Pi}^{-} k^{m}, P i^{-}\right.$ $s h]$ 福锦 $\left[\mathrm{Pi}^{-} z, P i^{-} s h\right] 、 \mathrm{~K} 1\left[P i^{-} t a\right] 、 \mathrm{Pi} 4$ 号 $\left[P i^{-} t a^{2}\right.$, $P i-s h]$ 砦 1 号 $\left[P^{-} z^{t}, P i-s h\right] 、 K 60\left[P^{-} k^{p}, P i-s h\right]$ 、 BL1 [ $\left.P i^{-} b, P i^{-} s h\right] 、$ K59 [ $\left.P i^{-} t, P i^{-} k^{s}\right]$ 和 K3 [ $\left.\mathrm{Pi}^{-} k^{h}\right]$; 80 个江苏省主栽品种和新育成品种。育苗 在 60 $\mathrm{cm} \times 30 \mathrm{~cm} \times 4 \mathrm{~cm}$ 的育秧盘内进行, 13 个已知抗病 基因品种,每盘播 3 行,每行播 9 个品种 (重复 2 次）, 每穴播 $15 \sim 20$ 粒种子, 以我国的普感品种丽江 新团黑谷作对照。江苏省主栽和新育成品种每盘播 3 行,每行播 9 个品种 (重复 2 次), 每穴播 $15 \sim 20$ 粒种子, 以我国的普感品种丽江新团黑谷作对照。2 叶期施硫胺一次, 4 叶期接种。

\section{4 接种和调查}

将 4 叶期的秧苗置于密封的玻璃接种箱 $(70 \mathrm{~cm}$ $\times 45 \mathrm{~cm} \times 33 \mathrm{~cm})$ 内, 在 $25^{\circ} \mathrm{C}$ 恒温室中通过接种箱 上的小圆孔用稻瘟病菌狍子悬浮液喷雾接种。接种 前先喷水, 使箱内湿度达 $100 \%$ 。接种后将秧苗在 接种箱中放置 $24 \mathrm{~h}$ 再移至遮荫棚内, 于白天每隔 1 $\mathrm{h}$ 定时喷水 $5 \mathrm{~min}$ 保湿。接种后 $7 \sim 8$ 天待其充分发 病, 按照目前普遍采用的标准 (Leung et al.,1988) 调查各品种的发病情况。按 13 个已知抗病基因品 种对供试菌株的抗感反应划分毒性类型。毒性类型 相似率分析按照朱衡等 (1991) 的方法, 计算公式: $S x y=[2 N x y /(N x+N y)] \times 100 \%$, 其中 $N x$ 和 $N y$ 分别代表 1 年及 1 个地区毒性类型的总数, $N x y$ 
代表它们相同的毒性类型数量。

\section{2 结果与分析}

\section{1 江苏省稻瘟病菌毒性的多样性及分布}

$1997 \sim 1999$ 年从江苏省采集的 324 个菌株,根

据其在 13 个已知抗病基因品种上的致病/非致病表 型,可划分为 90 种毒性类型 (表 1), 表明江苏省稻
瘟病菌存在着丰富的毒性多样性。其中 1997 年江 苏省的 109 个菌株中出现 47 种毒性类型,1998 年 在 109 个菌株中出现 35 种毒性类型, 1999 年在 106 个菌株中出现 37 种毒性类型 (表 2); 在 90 种毒性 类型中, 出现 10 次以上的毒性类型只有 6 种, 有 53 种毒性类型只出现 1 次 (表 1)。江苏省稻瘟病菌的 毒性类型在不同地区间和年度间存在着较大的差

表 $11997 \sim 1999$ 年江苏省稻瘟病菌的毒性类型及其分布

Table 1 Pathotypes of Magnaporthe grisea and their distribution in Jiangsu Province in 1997 1999

\begin{tabular}{|c|c|c|c|c|c|c|c|c|c|c|c|c|c|c|c|}
\hline \multirow{2}{*}{$\begin{array}{c}\text { 毒性类型 } \\
\text { 编号 } \\
\text { Pathotype }\end{array}$} & \multicolumn{13}{|c|}{ 毒性表型 Avirulence/ virulence to cultivars } & \multirow{2}{*}{$\begin{array}{c}\text { 分布地区及出现数量 } \\
\text { Area and isolate number of } \\
\text { the pathotype }\end{array}$} & \multirow{2}{*}{$\begin{array}{l}\text { 出现的年度 } \\
\text { Year detected }\end{array}$} \\
\hline & 1 & 2 & 3 & 4 & 5 & 6 & 7 & 8 & 9 & 10 & 11 & 12 & 13 & & \\
\hline 1 & 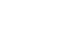 & $\bigcirc$ & $\bigcirc$ & $\bigcirc$ & $\bigcirc$ & $\mathrm{O}$ & & & $\bigcirc$ & O & $\mathrm{O}$ & 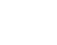 & $\bigcirc$ & $\mathrm{WJ}(4), \mathrm{TZ}(11), \mathrm{GYU}(10), \mathrm{GYO}(13), \mathrm{YX}(25)$ & $1997,1998,1999$ \\
\hline 2 & & ? & $\bigcirc$ & $\bigcirc$ & $\bigcirc$ & $\bigcirc$ & & & $\bigcirc$ & $\mathrm{O}$ & $\bigcirc$ & 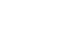 & $\bigcirc$ & WJ (1), TZ(3), GYU(1), GYO(5), YX(5) & $1997,1998,1999$ \\
\hline 3 & & & $\bigcirc$ & $\bigcirc$ & $\bigcirc$ & $\mathrm{O}$ & C & & $\bigcirc$ & $\mathrm{O}$ & $\mathrm{O}$ & $\bigcirc$ & $\bigcirc$ & $\mathrm{WJ}(2), \mathrm{TZ}(2), \mathrm{GYU}(7), \mathrm{GYO}(4), \mathrm{YX}(6)$ & $1997,1998,1999$ \\
\hline 4 & & $\bigcirc$ & $\bigcirc$ & $\bigcirc$ & $\bigcirc$ & O & $\bigcirc$ & $\bigcirc$ & $\bigcirc$ & $\mathrm{O}$ & $\mathrm{O}$ & $\bigcirc$ & $\bigcirc$ & $\mathrm{WJ}(3), \mathrm{TZ}(2), \mathrm{GYU}(3), \mathrm{GYO}(6), \mathrm{YX}(1)$ & $1997,1998,1999$ \\
\hline 5 & & $\bigcirc$ & $\bigcirc$ & $\bigcirc$ & $\bigcirc$ & $\mathrm{O}$ & & & $\bigcirc$ & 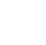 & $\mathrm{O}$ & $\bigcirc$ & $\bigcirc$ & WJ (2), GYU (1), GYO(1), YX(3) & 1998,1999 \\
\hline 6 & & $\bigcirc$ & $\bigcirc$ & $\bigcirc$ & $\bigcirc$ & $\mathrm{O}$ & & & $\mathrm{O}$ & $\bigcirc$ & $\bigcirc$ & & $\bigcirc$ & $\mathrm{TZ}(9), \mathrm{GYU}(9), \mathrm{GYO}(2), \mathrm{YX}(3)$ & $1997,1998,1999$ \\
\hline 7 & 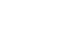 & O & $\bigcirc$ & $\bigcirc$ & $\bigcirc$ & O & & & $\bigcirc$ & $\bigcirc$ & $\mathrm{O}$ & $\bigcirc$ & $\bigcirc$ & $\mathrm{WJ}(1), \mathrm{TZ}(8), \mathrm{GYO}(1), \mathrm{YX}(1)$ & $1997,1998,1999$ \\
\hline 8 & $\bigcirc$ & $\bigcirc$ & $\bigcirc$ & $\bigcirc$ & $\bigcirc$ & $\mathrm{O}$ & $U$ & 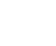 & $\bigcirc$ & $\mathrm{O}$ & $\mathrm{O}$ & $\bigcirc$ & $\bigcirc$ & $\mathrm{WJ}(3), \mathrm{TZ}(1), \mathrm{GYO}(2)$ & $1997,1998,1999$ \\
\hline 9 & & ○ & $\bigcirc$ & $\bigcirc$ & $\bigcirc$ & $\mathrm{O}$ & $\bigcirc$ & & 0 & $\mathrm{O}$ & $\mathrm{O}$ & $\bigcirc$ & $\bigcirc$ & $\mathrm{WJ}(5), \mathrm{TZ}(2), \mathrm{YX}(2)$ & $1997,1998,1999$ \\
\hline 10 & & 0 & $\bigcirc$ & $\bigcirc$ & $\bigcirc$ & $\bigcirc$ & 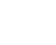 & & $\mathrm{O}$ & $\mathrm{O}$ & $\bigcirc$ & & $\bigcirc$ & $\mathrm{WJ}(2), \mathrm{GYO}(1), \mathrm{YX}(2)$ & 1997 \\
\hline 11 & & $\bigcirc$ & $\bigcirc$ & $\bigcirc$ & $\bigcirc$ & O & & & $\bigcirc$ & $\mathrm{O}$ & O & $\bigcirc$ & $\bigcirc$ & $\mathrm{GYO}(1), \mathrm{YX}(1)$ & 1999 \\
\hline 12 & $\bigcirc$ & $\bigcirc$ & $\bigcirc$ & $\bigcirc$ & 0 & $\mathrm{O}$ & 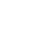 & 0 & $\bigcirc$ & $\mathrm{O}$ & $\mathrm{O}$ & $\bigcirc$ & $\bigcirc$ & GYU(3), GYO(2) & 1998,1999 \\
\hline 13 & & $\bigcirc$ & $\bigcirc$ & $\bigcirc$ & 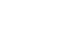 & $\mathrm{O}$ & & & $\bigcirc$ & $\bigcirc$ & $\mathrm{O}$ & $\bigcirc$ & $\bigcirc$ & GYU (2), GYO(6) & 1998,1999 \\
\hline 14 & & $\bigcirc$ & $\bigcirc$ & O & O & $\mathrm{O}$ & & 0 & $\bigcirc$ & & $\mathrm{O}$ & $\bigcirc$ & 0 & $\mathrm{WJ}(3), \mathrm{GYO}(1)$ & 1998,1999 \\
\hline 15 & & O & $\bigcirc$ & $\bigcirc$ & $\bigcirc$ & $\mathrm{O}$ & & & $\bigcirc$ & & $\mathrm{O}$ & $\bigcirc$ & $\bigcirc$ & $\mathrm{WJ}(1), \mathrm{TZ}(2)$ & 1998,1999 \\
\hline 16 & & $\bigcirc$ & $\bigcirc$ & $\bigcirc$ & $\bigcirc$ & $\mathrm{O}$ & & & $\bigcirc$ & & . & $\bigcirc$ & $\bigcirc$ & WJ (1), YX(1) & 1999 \\
\hline 17 & & $\bigcirc$ & $\bigcirc$ & O & 0 & $\mathrm{O}$ & & D & $\bigcirc$ & 0 & $\mathrm{O}$ & 0 & 0 & WJ (1), GYU (1) & 1999 \\
\hline 18 & & $\bigcirc$ & $\bigcirc$ & $\bigcirc$ & 8 & $\mathrm{O}$ & & & $\bigcirc$ & 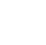 & $\mathrm{O}$ & $\bigcirc$ & $\bigcirc$ & GYU (2), GYO(2) & 1999 \\
\hline 19 & $\bigcirc$ & ? & $\bigcirc$ & $\bigcirc$ & $\bigcirc$ & $\mathrm{O}$ & $\bigcirc$ & $\bigcirc$ & $\bigcirc$ & $\mathrm{O}$ & $\mathrm{O}$ & $\bigcirc$ & $\bigcirc$ & WJ (1), GYO (1) & 1997 \\
\hline 20 & & 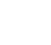 & $\bigcirc$ & $\bigcirc$ & $\bigcirc$ & O & $\bigcirc$ & $\bigcirc$ & $\bigcirc$ & $\bigcirc$ & O & $\bigcirc$ & $\bigcirc$ & $\mathrm{WJ}(7), \mathrm{TZ}(1)$ & $1997,1998,1999$ \\
\hline 21 & & 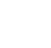 & 0 & $\bigcirc$ & $\bigcirc$ & $\mathrm{O}$ & & & 0 & $\mathrm{O}$ & $\mathrm{O}$ & & $\bigcirc$ & $\mathrm{WJ}(2), \mathrm{TZ}(1)$ & 1997,1998 \\
\hline 22 & $U$ & 0 & 0 & 0 & 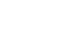 & ? & & 0 & $\mathrm{O}$ & $\bigcirc$ & $\mathrm{O}$ & ( & 0 & GYU(1), GYO(2) & 1997 \\
\hline 23 & & $\bigcirc$ & $\bigcirc$ & $\bigcirc$ & $\bigcirc$ & $\mathrm{O}$ & & & $\bigcirc$ & $\bigcirc$ & $\mathrm{O}$ & & 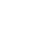 & GYU (1), YX(1) & 1997,1999 \\
\hline 24 & & $\bigcirc$ & $\bigcirc$ & $\bigcirc$ & $\bigcirc$ & O & & & $\bigcirc$ & & $\bigcirc$ & & $\bigcirc$ & $\mathrm{TZ}(2), \mathrm{GYU}(1)$ & 1997 \\
\hline 25 & & 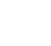 & $\bigcirc$ & $\bigcirc$ & $\bigcirc$ & $\mathrm{O}$ & O & $\bigcirc$ & $\bigcirc$ & $\bigcirc$ & $\bigcirc$ & & $\bigcirc$ & GYU (1), YX(1) & 1997 \\
\hline 26 & & ? & $\bigcirc$ & $\bigcirc$ & $\bigcirc$ & O & & & $\bigcirc$ & $\bigcirc$ & & & $\bigcirc$ & $\mathrm{GYO}(1), \mathrm{YX}(5)$ & 1997 \\
\hline 27 & & $\bigcirc$ & $\bigcirc$ & $\bigcirc$ & $\bigcirc$ & 0 & 0 & 0 & $\bigcirc$ & $\mathrm{O}$ & $\mathrm{O}$ & 0 & $\bigcirc$ & $\mathrm{TZ}(1), \mathrm{GYO}(1)$ & 1997,1998 \\
\hline 28 & & $\bigcirc$ & $\bigcirc$ & $\bigcirc$ & $\bigcirc$ & O & & & $\bigcirc$ & $\bigcirc$ & 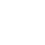 & C & $\bigcirc$ & $\mathrm{TZ}(1), \mathrm{GYO}(1)$ & 1997,1999 \\
\hline 29 & & $\bigcirc$ & $\bigcirc$ & 0 & 0 & 0 & & & $\mathrm{O}$ & O & O & $\bigcirc$ & $\bigcirc$ & WJ (4) & 1998,1999 \\
\hline 30 & & $\bigcirc$ & $\bigcirc$ & 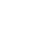 & 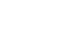 & $\mathrm{O}$ & & & $\bigcirc$ & & $\bigcirc$ & $\mathrm{O}$ & & WJ (7) & 1998,1999 \\
\hline $31 \sim 90$ * & & & & & & & & & & & & & & & \\
\hline$\sum \mathrm{I}_{\mathrm{i}}{ }^{* *}$ & 301 & 98 & 23 & 50 & 76 & 26 & 215 & 233 & 1 & 57 & 25 & 95 & 32 & & \\
\hline
\end{tabular}

表中 毒性表型”一栏中阿拉伯数字 1 13 分别代表品种: $1=$ 新 2 号 [Pi- $\left.k^{s}, P i-s h\right] ; 2=$ 爱知旭 $\left[P i^{-} a, P i-19(t)\right] ; 3=$ 藤坂 5 号 $[P i-i, P i-s h] ; 4$ =草笛 $\left[P i^{-} k, P i^{-} s h\right] ; 5=$ 露明 $\left[P i^{-} k^{m}, P i^{-} s h\right] ; 6=$ 福锦 $\left[P i^{-} z, P i^{-} s h\right] ; 7=\mathrm{K} 1\left[P i^{-} t a\right] ; 8=\mathrm{Pi} 4$ 号 $\left[P i^{-} t a^{2}, P i^{-} s h\right] ; 9=$ 砦 1 号 $\left[P i^{-} z^{t}, P i^{-} s h\right] ; 10=$ K60 [ $\left.P i^{-} k^{p}, P i-s h\right] ; 11=\mathrm{BL} 1\left[\mathrm{Pi}^{-} b, \mathrm{Pi}^{-} \mathrm{sh}\right] ; 12=\mathrm{K} 59\left[\mathrm{Pi}^{-} t, \mathrm{Pi}^{-} k^{s}\right] ; 13=\mathrm{K} 3\left[\mathrm{Pi}^{-} k^{h}\right]$ 。

表示致病; 表示不致病。WJ $=$ 吴江; $\mathrm{TZ}=$ 通州; $\mathrm{GYU}=$ 赣榆; $\mathrm{GYO}=$ 高邮; $\mathrm{YX}=$ 宜兴。* : 毒性类型 $31 \sim 90$ 仅在 1 个地区和 1 个年度 出现,其中 1 种毒性类型出现 4 次, 1 种毒性类型出现 3 次, 5 种毒性类型出现 2 次。** $\Sigma \mathrm{I}_{\mathrm{i}}$ 表示能侵染各品种的菌株数量。

Note: $1=$ Shin2, $2=$ Aich Asahi , $3=$ Fujisaka, $4=$ Kusabue, $5=$ Tsuyuake, $6=$ Fuku-nishiki , $7=$ K1 , $8=$ PiNo. $4,9=$ Toride1, $10=$ K60 , $11=$ $\mathrm{BL} 1,12=\mathrm{K} 59,13=\mathrm{K} 3$.

Virulent, OAvirulent; WJ ,TZ,GYU, GYO and YX stand for Wujiang, Tongzhou, Ganyu, Gaoyou and Yixing, respectively. * Pathotype 31 90 only appeared in one area and one year. Among them one pathotype appeared four times, one pathotype appeared three times and five pathotypes appeared one time. $* * \Sigma \mathrm{I}_{\mathrm{I}}$ stand for virulence isolate number to the cultivar. 
异:有 62 种毒性类型只在 1 个地区出现, 有 18 种在 2 个地区出现, 在 3 个地区和 4 个地区出现的各有 3 种,在 5 个地区均出现的仅有 4 种类型; 在 3 年中 均出现的毒性类型只有 9 种, 2 年中均出现的毒性 类型有 11 种,其余只在某一年份出现 (表 1)。不同 地区毒性类型组成年度间的变化亦有差异 (表 1 , 表 2)。通州地区变化较大, 毒性类型数量逐年增加, 毒 性组成趋向复杂; 宜兴地区的毒性类型组成则一直 较简单并且存在优势毒性类型; 吴江地区的毒性类 型组成在 3 年中均较复杂; 赣榆地区的毒性类型组 成则由复杂到简单。

2.2 江苏省稻瘟病菌对 13 个已知抗病基因品种的 毒力测定

根据 1997 1999 年江苏省稻瘟病菌菌株对 13 个日本已知抗病基因品种的致病性分析表明 (表 1) ,江苏省稻瘟病菌对品种新 2 号、 $\mathrm{K} 1$ 和 $\mathrm{Pi} 4$ 号（携 带的已知抗病基因分别为 $\mathrm{Pi}^{-} k^{s} / \mathrm{Pi}^{-} s h 、 \mathrm{Pi}^{-} \mathrm{ta}$ 和 $\mathrm{Pi}^{-}$ $\left.t a^{2} / P i-s h\right)$ 具有较高的侵染率, 324 个菌株中分别有 301 个、215 个和 233 个菌株能侵染上述品种, 即有 $66.4 \% \sim 92.9 \%$ 的供试菌株可以克服上述品种的抗 性从而对上述抗病基因品种表现出具有毒性。对品 种藤坂 5 号、福锦、些 1 号和 BL1 (抗病基因分别为

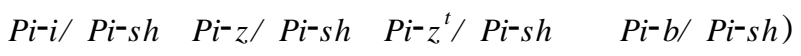
侵染率较低, 分别只有 23 个、26个、1 个和 25 个菌 株能侵染这些品种。上述结果表明, 对江苏省的稻 瘟病菌, $P i^{-} k^{s} 、 P i^{-} t a 、 P i^{-} t a^{2} 、 P i^{-} s h$ 这些基因的抗谱 很窄, 而 $P i^{-} i 、 P i^{-} z 、 P i^{-} z^{t}$ 和 $P i^{-} b$ 等基因具有很宽的
抗谱,可作为抗源加以利用。

2.3 江苏省稻瘟病菌毒性类型组成的地区间差异 及变化趋势

江苏省稻瘟病菌的毒性类型组成在年度间有一 定差异,表现多变和不稳定,且随着时间的推移有差 异加大的趋势。对江苏省及 5 个代表地区年度间稻 瘟病菌毒性类型组成的相似性分析结果 (表 3) 显 示, 全省 1997 年与 1998 年间毒性类型的相似率为 $26.83 \%, 1998$ 年与 1999 年的相似率为 $44.44 \%$, 而 1997 年与 1999 年的相似率仅 $21.43 \%$; 江苏省 5 个 代表地区年度间毒性类型相似率的变化亦反映了相 同的趋势。上述变化不仅反映出年度间毒性类型数 量上的不同, 同时反映出毒性组成 (种类) 存在很大 的差异。地区间稻瘟病菌的毒性类型组成亦存在较 大的差异, 对 5 个代表地区间的相似性分析结果 (表 4) 显示, 相似率范围从 $6.25 \%$ 到 $58.52 \%$ 。赣榆、高 邮和宜兴这 3 个地区菌株的毒性类型相似率较高, 3 年平均相似率在 $35.5 \% \sim 44.86 \%$ 之间; 吴江和通 州与上述 3 个地区菌株毒性类型差异较大, 相似率 较低;而吴江与通州的菌株之间的毒性类型差异亦 很大, 平均相似率只有 $18.12 \%$ 。

2.4 江苏省水稻主栽品种和新育成品种对江苏省 稻瘟病菌的抗病性分析

从江苏省 5 个代表地区分离到的稻瘟病菌菌株 中选择 6 种毒性类型的菌株 (每一毒性类型各选一 个代表菌株）,其致病谱覆盖了所有 13 个已知抗病 基因品种,并且包含了江苏省的主要小种(表 5)。

表 2 江苏省 5 个代表地区 $1997 \sim 1999$ 年稻瘟病菌毒性类型出现的数量

Table 2 Number of pathotypes of Magnaporthe grisea in five typical areas of Jiangsu Province in $1997 \sim 1999$

\begin{tabular}{ccccccc}
\hline 年度 & \multicolumn{5}{c}{ 毒性类型的数量 Number of pathotypes } \\
\cline { 2 - 7 } Year & ${\text { 江苏省 }{ }^{*} \text { Jiangsu }}^{\text {吴江 Wujiang }}$ & 赣榆 Ganyu & 通州 Tongzhou & 高邮 Gaoyou & 宜兴 Yixing \\
\hline 1997 & 47 & 13 & 19 & 8 & 14 & 9 \\
1998 & 35 & 15 & 7 & 15 & 10 & 7 \\
1999 & 37 & 13 & 10 & 20 & 11 & 7 \\
\hline
\end{tabular}

*江苏省稻瘟病菌毒性类型数量是上述 5 个地区各年度出现的毒性类型数量的合计。

* The pathotype number of Magnaporthe grisea in Jiangsu Province is the annual total amount of pathotype number detected in the five typical areas.

表 $31997 \sim 1999$ 年江苏省及 5 个代表地区年度间稻瘟病菌毒性类型的相似率

Table 3 Similarity of pathotypes between different years in five typical areas of Jiangsu Province in 1997 1999

\begin{tabular}{ccccccc}
\hline 年度间 & \multicolumn{5}{c}{ 相似率 $(\%)$ Similarity of pathotypes } \\
\cline { 2 - 7 } Years compared & ${\text { 江苏省 }{ }^{*} \text { Jiangsu }}^{\text {吴江 Wujiang }}$ & 通州 Tongzhou & 高邮 Gaoyou & 赣榆 Ganyu & 宜兴 Yixing \\
\hline $1997 / 1998$ & 26.83 & 21.43 & 26.09 & 0 & 20.69 & 25 \\
$1998 / 1999$ & 44.44 & 28.57 & 45.71 & 47.62 & 47.06 & 42.86 \\
$1997 / 1999$ & 21.43 & 7.69 & 21.43 & 0 & 13.79 & 12.5 \\
\hline
\end{tabular}

*注文同于表 2 。

* See note of table 2. 
表 $41997 \sim 1999$ 年江苏省不同地区间稻瘟病菌毒性类型的相似率

Table 4 Similarity of pathotypes between each two of five typical areas of Jiangsu Province in 1997 1999

\begin{tabular}{|c|c|c|c|c|c|c|c|c|c|c|}
\hline \multirow[b]{2}{*}{$\begin{array}{l}\text { 年度 } \\
\text { Year }\end{array}$} & \multicolumn{10}{|c|}{ 相似率 (\%) Similarity of pathotypes } \\
\hline & $\begin{array}{c}\text { 吴江/ 通州 } \\
\text { Wujiang/ } \\
\text { Tongzhou }\end{array}$ & $\begin{array}{c}\text { 吴江/ 赣榆 } \\
\text { Wujiang/ } \\
\text { Ganyu }\end{array}$ & $\begin{array}{c}\text { 吴江/ 宜兴 } \\
\text { Wujiang/ } \\
\text { Yixing } \\
\end{array}$ & $\begin{array}{c}\text { 吴江/ 高邮 } \\
\text { Wujiang/ } \\
\text { Gaoyou }\end{array}$ & $\begin{array}{c}\text { 通州/赣榆 } \\
\text { Tongzhou/ } \\
\text { Ganyu }\end{array}$ & $\begin{array}{c}\text { 通州/高邮 } \\
\text { Tongzhou/ } \\
\text { Gaoyou }\end{array}$ & $\begin{array}{c}\text { 通州/宜兴 } \\
\text { Tongzhou/ } \\
\text { Yixing }\end{array}$ & $\begin{array}{c}\text { 赣榆/高邮 } \\
\text { Ganyu/ } \\
\text { Gaoyou }\end{array}$ & $\begin{array}{c}\text { 赣榆/ 宜兴 } \\
\text { Ganyu/ } \\
\text { Yixing } \\
\end{array}$ & $\begin{array}{c}\text { 高邮/ 宜兴 } \\
\text { Gaoyou/ } \\
\text { Yixing } \\
\end{array}$ \\
\hline 1997 & 9.52 & 6.25 & 9.09 & 22.22 & 29.63 & 9.09 & 23.53 & 12.12 & 28.57 & 26.09 \\
\hline 1998 & 26.67 & 27.27 & 36.36 & 32.0 & 36.36 & 32.0 & 36.36 & 58.82 & 57.14 & 47.06 \\
\hline 1999 & 18.18 & 26.09 & 30.0 & 33.33 & 20.0 & 25.81 & 14.81 & 63.64 & 23.53 & 33.33 \\
\hline $\begin{array}{c}\text { 平均 } \\
\text { Average }\end{array}$ & 18.12 & 19.87 & 25.15 & 29.18 & 28.66 & 22.30 & 24.90 & 44.86 & 36.41 & 35.50 \\
\hline
\end{tabular}

表 5 选用的江苏省 6 个代表性毒性类型在 13 个已知抗性基因品种上的致病表型 *

Table 5 Six typical pathotypes of Jiangsu Province and their virulence on 13 cultivars which resistance genes have been determined

\begin{tabular}{|c|c|c|c|c|c|c|c|c|c|c|c|c|c|c|c|}
\hline \multirow{2}{*}{$\begin{array}{l}\text { 毒性类型 } \\
\text { Pathotype }\end{array}$} & \multirow{2}{*}{$\begin{array}{l}\text { 采集地 } \\
\text { Locality }\end{array}$} & \multirow{2}{*}{$\begin{array}{c}\text { 小种 }{ }^{* *} \\
\text { Race }\end{array}$} & \multicolumn{13}{|c|}{$\begin{array}{c}\text { 在 } 13 \text { 个已知抗性基因品种上的致病表型 }{ }^{* * *} \\
\text { Virulence on } 13 \text { cultivars which resistance genes have been determined }\end{array}$} \\
\hline & & & 1 & 2 & 3 & 4 & 5 & 6 & 7 & 8 & 9 & 10 & 11 & 12 & 13 \\
\hline $\begin{array}{l}\text { Pathotype } \\
\quad 50\end{array}$ & $\begin{array}{l}\text { 通州 } \\
\text { Tongzhou }\end{array}$ & $\mathrm{ZB}_{5}$ & $\bullet$ & $\bullet$ & 0 & 0 & 0 & 0 & 0 & $\bigcirc$ & 0 & 0 & 0 & 0 & 0 \\
\hline $\begin{array}{l}\text { Pathotype } \\
\quad 37\end{array}$ & $\begin{array}{c}\text { 吴江 } \\
\text { Wujiang }\end{array}$ & $\mathrm{ZD}_{1}$ & $\bullet$ & $\bullet$ & 0 & 0 & • & • & 0 & $\bullet$ & 0 & • & 0 & • & $\bigcirc$ \\
\hline $\begin{array}{c}\text { Pathotype } \\
41\end{array}$ & $\begin{array}{l}\text { 赣榆 } \\
\text { Ganyu }\end{array}$ & $\mathrm{ZE}_{3}$ & $\bullet$ & $\bigcirc$ & $\bigcirc$ & $\bullet$ & $\bullet$ & $\bullet$ & $\odot$ & $\bigcirc$ & 0 & $\bullet$ & $\bigcirc$ & $\bullet$ & $\bullet$ \\
\hline $\begin{array}{c}\text { Pathotype } \\
6\end{array}$ & $\begin{array}{l}\text { 赣榆 } \\
\text { Ganyu }\end{array}$ & $\mathrm{ZF}_{1}$ & $\bullet$ & 0 & 0 & 0 & $\bigcirc$ & $\bigcirc$ & 0 & $\bullet$ & 0 & 0 & $\bigcirc$ & • & $\bigcirc$ \\
\hline $\begin{array}{c}\text { Pathotype } \\
2\end{array}$ & $\begin{array}{c}\text { 高邮 } \\
\text { Gaoyou }\end{array}$ & $\mathrm{ZG}_{1}$ & 0 & $\bullet$ & 0 & 0 & $\bigcirc$ & $\bigcirc$ & 0 & 0 & 0 & 0 & 0 & 0 & 0 \\
\hline $\begin{array}{c}\text { Pathotype } \\
\quad 48\end{array}$ & $\begin{array}{c}\text { 通州 } \\
\text { Tongzhou }\end{array}$ & $\mathrm{ZG}_{1}$ & 0 & 0 & 0 & 0 & 0 & 0 & 0 & 0 & 0 & $\bigcirc$ & $\bigcirc$ & 0 & $\bigcirc$ \\
\hline
\end{tabular}

表中 致病表型”一栏中阿拉伯数字 $1 \sim 13$ 分别代表品种: $1=$ 新 2 号 $\left[P i^{-} k^{s}, P i-s h\right] ; 2=$ 爱知旭 $\left[P i^{-} a, P i-19(t)\right] ; 3=$ 藤坂 5 号 $[P i-i, P i-s h] ; 4$ = 草笛 $\left[P i^{-} k, P i-s h\right] ; 5=$ 露明 $\left[P i^{-} k^{m}, P i-s h\right] ; 6=$ 福锦 $\left[P i^{-} z, P i-s h\right] ; 7=\mathrm{K} 1\left[P i^{-} t a\right] ; 8=\mathrm{Pi} 4$ 号 $\left[P i^{-} t a^{2}, P i^{-} s h\right] ; 9=$ 砦 1 号 $\left[P i^{-} z^{t}, P i^{-} s h\right] ; 10=$ K60[ $\left.P i^{-} k^{p}, P i-s h\right] ; 11=\mathrm{BL} 1\left[P i^{-} b, P i-s h\right] ; 12=\mathrm{K} 59\left[P i^{-} t, P i-k^{s}\right] ; 13=\mathrm{K} 3\left[P^{-}{ }^{-} k^{h}\right] 。$

**:各毒性类型菌株在中国统一的 7 个鉴别品种上表现出的小种类型。***: 表示致病; $\bigcirc$ 表示不致病。

Note: $1=$ Shin2, $2=$ Aich Asahi , $3=$ Fujisaka, $4=$ Kusabue, $5=$ Tsuyuake, $6=$ Fuku-nishiki , $7=\mathrm{K} 1,8=$ PiNo. $4,9=$ Toride1, $10=\mathrm{K} 60,11=$ $\mathrm{BL} 1,12=\mathrm{K} 59,13=\mathrm{K} 3$.

** The races determined when the selected pathotypes were assessed on seven Chinese differential cultivars. *** Virulence; OAvirulence.

表 6 江苏省主栽和新育成品种对江苏省稻瘟病菌 6 个代表毒性类型的抗性分析

Table 6 Analysis of resistance to six typical pathotypes of Magnaporthe grisea on major cultivars and newly-bred cultivars in Jiangsu Province

\begin{tabular}{|c|c|c|c|c|c|c|c|}
\hline \multirow{2}{*}{$\begin{array}{l}\text { 品种类型 } \\
\text { Cultivar type }\end{array}$} & \multirow{2}{*}{$\begin{array}{l}\text { 供试品种数量 } \\
\text { No. of cultivars } \\
\text { tested }\end{array}$} & \multicolumn{6}{|c|}{$\begin{array}{c}\text { 供试品种对 } 6 \text { 个毒性类型感病率 }(\%) \\
\text { Frequency of susceptability of different types of cultivar to six pathotypes }\end{array}$} \\
\hline & & $\begin{array}{c}\text { Pathotype } \\
37\end{array}$ & $\begin{array}{c}\text { Pathotype } \\
41\end{array}$ & $\begin{array}{c}\text { Pathotype } \\
50\end{array}$ & $\begin{array}{c}\text { Pathotype } \\
2 \\
\end{array}$ & $\begin{array}{l}\text { Pathotype } \\
48\end{array}$ & $\begin{array}{c}\text { Pathotype } \\
6\end{array}$ \\
\hline $\begin{array}{l}\text { 常规中籼 } \\
\text { Indica }\end{array}$ & 8 & 0 & 0 & 0 & 0 & 0 & 0 \\
\hline $\begin{array}{l}\text { 杂交中籼 } \\
\text { Indica hybrid }\end{array}$ & 11 & 0 & 0 & 0 & 0 & 0 & 0 \\
\hline $\begin{array}{c}\text { 中熟中粳 } \\
\text { Medium japonica }\end{array}$ & 16 & 31.25 & 25 & 43.75 & 56.25 & 50 & 56.25 \\
\hline $\begin{array}{c}\text { 迟熟中粳 } \\
\text { Late medium japonica }\end{array}$ & 15 & 6.67 & 26.67 & 26.67 & 60 & 53.33 & 60 \\
\hline $\begin{array}{c}\text { 杂交中粳 } \\
\text { Medium japonica hybrid }\end{array}$ & 11 & 0 & 0 & 27.27 & 0 & 0 & 0 \\
\hline $\begin{array}{l}\text { 杂交晚粳 } \\
\text { Late japonica hybrid }\end{array}$ & 8 & 0 & 0 & 37.5 & 0 & 0 & 0 \\
\hline $\begin{array}{c}\text { 单季晚粳 } \\
\text { Late japonica }\end{array}$ & 11 & 9.09 & 9.09 & 36.36 & 36.36 & 36.36 & 36.36 \\
\hline
\end{tabular}


用上述 6 个毒性类型的菌株对 80 个江苏省主栽和 新育成品种进行接种, 结果 (表 6) 显示, 所有常规中 籼稻和杂交中籼稻对 6 个毒性类型均表现为抗病, 表现出对江苏省稻瘟病菌具广谱抗性; 杂交中粳和 杂交晚粳只对毒性类型 50 感病, 对其他 5 种毒性类 型均表现抗病, 抗性谱较广; 而常规中、晚粳类品种 对 6 个代表毒性类型均有一定程度的感病, 其中, 参 试的 42 个常规粳稻 (包括中熟中粳, 迟熟中粳和单 季晚粳) 品种中, 有 10 个品种对供试 6 个毒性类型 均表现为抗病,占 $23.8 \%$,有 3 个品种均表现为感 病, 占 $7.1 \%, 9$ 个品种只对其中的 1 种毒性类型感 病, 占 $21.4 \%, 16$ 个品种分别对 $3 \sim 5$ 个毒性类型感 病, 占 $38.1 \%$ 。在所有参试品种中, 中熟中粳和迟 熟中粳的感病率最高 (表 6), 表明这类品种对江苏 省稻瘟病菌的抗性较差。上述品种中所包含的抗病 基因还有待进一步研究来明确。

\section{3 讨论}

本研究表明江苏省稻瘟病菌存在着丰富的毒性 多样性,毒性类型组成在地区间存在较大的差异, 在 年度间亦有明显变化, 呈现出多变、不稳定的特征, 表明稻瘟病菌在遗传上存在着很大的毒性变化空 间, 可能是导致抗病品种抗病性丧失的主要原因之

利用作物种内具不同基因型品种的合理布局， 发挥作物遗传多样性对病害的控制效果, 可以减缓 混合栽培区病害的流行速度 (Mundt \& Browning, 1985 ; Wolfe ,1985)。Koizumi (1994) 用水稻抗病品 种和感病品种混合栽培大大降低了感病品种稻瘟病 的发病率。云南农业大学的研究表明, 在遗传背景 差异较大的水稻品种混栽的田块中, 虽然稻瘟病菌 毒性组成复杂, 但没有明显的优势组群, 而在单一品 种种植的田块, 其病菌毒性组成较为简单, 且有明显 的优势组群。他们利用遗传背景差异较大的品种混 合栽培, 大大降低了感病的糯稻品种的发病程度 ${ }^{1}$ 。 江苏的吴江市历史上是稻瘟病的高发区, 1994 年引 进广谱抗性品种 91-17 后, 又引进种植了 93-31、武 育粳 5 号、9522 等广谱抗性品种, 并逐渐形成了多 品种的搭配布局, 因而在 1994 年以后稻瘟病基本上
得到了控制(严大富等, 1999)。本研究表明, 江苏省 的籼稻品种和杂交稻品种对江苏省稻瘟病菌有很好 的抗性, 江苏省稻瘟病菌菌株对 $P i^{-} i 、 P i^{-} z, P i^{-} z^{t}$ 和 $P i^{-} b$ 等抗性基因的侵染率很低, 这些类型的品种和 抗性基因亦可以作为抗源加以利用。因此,在病害 严重地区和多发地区通过利用不同类型的品种合理 布局, 采用籼稻和粳稻、杂交稻和常规稻的合理搭 配,可望有效地控制稻瘟病的暴发流行。

\section{参考文献}

杜正文 (主编)，1991。中国水稻病虫害综合防治策略与技 术. 北京:农业出版社, $4 \sim 28$

严大富，花家禄，陆凡，王法明，杨金龙，陈以仁,1999. 吴江 稻区稻瘟病菌小种更替规律及抗瘟品种的利用. 江苏 农业学报, 15(3): 141 146

杨立昫, 崔继林, 汤玉庚(主编), 1990. 江苏稻作科学. 南京: 江苏科学技术出版社, 3

朱衡, 蒋琬如, 陈美玲, 王久林, 雷财林, 凌忠专, 朱立煌, 1991. 稻瘟病菌株的 DNA 指纹及其与小种致病性相互 关系的研究. 作物学报, 20(3):257 263

Koizumi S, 1994. Effect of field resistance on leaf blast dever opment in mixtures of susceptible and resistant rice cultivars. Annals of the Phytopathology Society of Japan, 60 : $585 \sim 594$

Leung H, Borromeo E S, Bernardo M A and Notteghem J L, 1988. Genetic analysis of virulence in the rice blast fungus Magnaporthe grisea. Phytopathology, 78:1227 1233

Mekwatanakarn P, Kositratana W, Levy M and Zeigler R S, 2000. Pathotype and avirulence gene diversity of Pyricularia grisea in Thailand as determined by rice Lines nearisogenic for major resistance genes. Plant Disease, 84:60 $\sim 70$

Mundt C C and Browning J A, 1985. Genetic diversity and cereal rust management. The Cereal Rust. Orlando: Academic Press ,2 : $527 \sim 560$

Ou S H, 1980. Pathogen variability and host resistance in rice blast disease. A nnual Review of Phytopathology, 18:167 $\sim 187$

Ou S H, 1985. Rice Diseases (2nd edition). Surrey, England: CMI press , $109 \sim 201$

Wolfe M S , 1985. The current status and prospects of multiline cultivars and variety mixtures for disease resistance. $A n^{-}$ nual Review of Phytopathology, $23: 251 \sim 273$

Zeigler R S, Leong S A and Teng P S, 1994. Rice Blast Disease. Wilmington, United Kingdom: CAB International, $267 \sim 292$ 